\title{
Upgrading Mice to Elephants: Effects and End-Point Solutions
}

\author{
Amit Mondal and Aleksandar Kuzmanovic \\ Department of Electrical Engineering and Computer Science \\ Northwestern University \\ Evanston, IL, 60208, USA \\ \{a-mondal, akuzma\}@cs.northwestern.edu
}

\begin{abstract}
Short TCP flows may suffer significant responsetime performance degradations during network congestion. Unfortunately, this creates an incentive for misbehavior by clients of interactive applications (e.g., gaming, telnet, web): to send "dummy" packets into the network at a TCP-fair rate even when they have no data to send, thus improving their performance in moments when they do have data to send. Even though no "law" is violated in this way, a large-scale deployment of such an approach has the potential to seriously jeopardize one of the core Internet's principles - statistical multiplexing. We quantify, by means of analytical modeling and simulation, gains achievable by the above misbehavior. Our research indicates that easy-to-implement application-level techniques are capable of dramatically reducing incentives for conducting the above transgressions, still without compromising the idea of statistical multiplexing.
\end{abstract}

Index Terms - Interactive application, TCP, retransmission timeout, statistical multiplexing

\section{INTRODUCTION}

It is well known that short TCP flows may experience significant performance degradations when they multiplex with long-lived TCP flows [2]. The root of the problem is the lack of knowledge about the level of the underlying network congestion. In absence of the large number of packets characteristic for long-lived flows, even a single packet loss can force a short-lived TCP flow to experience long retransmission timeouts [3], which in turn significantly increase a client's perceived response time. While several solutions have been proposed to efficiently combat the problem, none has been deployed in the Internet, probably because they require nonnegligible architectural changes [2], [4], [5].

However, one extremely relevant — and imminent — aspect of this problem is still unexplored. In essence, TCP-based interactive applications such as gaming [6], telnet, or persistent HTTP [7], which share the above problem common for short flows, have an incentive to improve their performance; still, without waiting for any Internet-wide architectural changes. In particular, they can "upgrade" themselves from "mice" to "elephants" in a trivial way, simply by sending packets into the network at a TCP-fair rate even when they have nothing to send. In this way, they become capable of developing larger congestion windows, avoid "losing memory" in moments

A subset of this work appears in the Proceedings of IEEE Infocom '07 [1]. of application-level data starvation [8], and improve their performance by avoiding long retransmission timeouts.

There are two reasons why users would want to reduce the response times. First, while the common wisdom is that $50-100 \mathrm{~ms}$ is the lower bound that clients care about; this is still an open research question. Recent results indicate that there is a tremendous variation in user satisfaction in interactive applications [9]. Second, there are scenarios, such as multiplayer games over the Internet, where users would care about improving their performance relative to other players, hence reducing their response time.

While it may appear that this is a minor problem, or even there is no problem at all (given that all flows are TCP friendly), this is far from being the case. While interactive flows account for a small fraction of the total Internet traffic in bytes, their percent in terms of the number of flows in the Internet is much higher [10], [11]. Moreover, interactive flows (e.g., chat and gaming) are long-lived in general. A large-scale deployment of this approach has the potential to seriously jeopardize one of the core principles that today's Internet is built upon — statistical multiplexing. Indeed, if everyone started taking their own fair bandwidth share, the network would soon become highly congested. While the absolute performance of all flows would necessarily degrade in such a case, a troubling observation is that those applying the fully-backlogged approach would still benefit relative to the regular clients. Hence, the dangerous incentive remains.

Unfortunately, upgrading an interactive to a fullybacklogged flow is easy to implement, both at the TCP and the application levels. Indeed, client-side only implementations could dramatically improve user-experienced response times, still without requiring any changes at servers. Moreover, inducing servers to send traffic at TCP-fair rates is not impossible [12]. In all scenarios, both network- and endpoint-based mechanisms that check for TCP-friendliness, e.g., [12]-[14], are incapable of detecting any violation, simply because all flows are TCP friendly.

To understand all aspects of the above problem, we conduct an extensive modeling and simulation analysis. By combining and extending the modeling results of [15]-[18], we quantify the response-time gains that fully-backlogged flows achieve over the interactive ones. Our results show that the expected response times of fully-backlogged flows can be two to three times smaller than those of interactive ones. Likewise, gains 
achievable by fully-backlogged TCP flows are much more pronounced in the case of Random Early Drop (RED) queues. Even if a packet is dropped at a RED bottleneck in the network, the probability is high that at least three of the followup packets will trigger the triple-duplicate ACK mechanism, thus avoiding long retransmission timeouts. Because Drop Tail queues induce correlated packet losses, the corresponding gain is smaller.

Further, we explore techniques that regular clients can apply to mitigate the problem. Given the inherent deployment issues with network-based solutions [2], [4], [5], we focus on endpoint based methods. We initially explore a TCP-level approach of reducing the retransmission timeout parameter by a half. Despite evident improvements, both our modeling and simulation results indicate that the method is incapable of removing the dangerous incentive for misbehavior.

We further explore two other endpoint techniques to address the problem: (i) short-term padding, and (ii) a diversity approach. In the first scenario, applications append a small number of small-sized packets to data bursts, thus increasing the probability to invoke the triple-duplicate ACK mechanism. In the second scenario, TCP endpoints repeat their packets: if at least one reaches the destination, the response time is small. Surprisingly, our modeling and simulation results indicate that neither approach is uniformly better, and in particular the impact of each strategy on system performance depends upon the queuing discipline (e.g., RED vs. Drop Tail) that is in use.

Finally, our results clearly show that both endpoint techniques outperform the fully-backlogged approach, thus effectively removing the dangerous incentive for the greedy TCPfriendly behavior. While various sub-versions of the proposed application-level techniques could themselves become attractive options for misbehaving clients, this no longer poses a threat to the Internet. Indeed, we show that even if all interactive-application clients deploy one of the proposed approaches, the overall network performance does not change dramatically. Thus, the statistical-multiplexing benefits remain available to all network clients.

The analysis and approaches presented in the paper are useful in cases where a single access link is shared among multiple end users. Because the bandwidth of the access link is limited, if a subset of the users adopt the fully-backlogged scheme, other users' transmission is severely affected. For example, many people working at home suffer from their housemates' greedy applications like BitTorrent and Flashget. Our analysis applies to such scenarios as well. The sustainable approaches we discuss towards the end of the paper can be used to improve the performance of the application-limited flows in presence of greedy cross traffic.

This paper is structured as follows. Section II explains the problem origins and its implications. In Section III, we quantify the padding-induced gain and present modeling and simulation results. In Section IV, we explore sustainable countermeasures, evaluate their performance, and compare overheads. We discuss related work in Section V. Finally, in Section VI we conclude.

\section{PRoblem ORIGINS AND IMPLICATIONS}

\section{A. Problem Origins}

TCP congestion control operates at two timescales. On smaller time scales of the order of RTTs, TCP performs additive-increase multiplicative-decrease (AIMD) control with the objective of having each flow transmit at the fair rate of its bottleneck link. At times of severe congestion in which multiple losses occur, TCP operates on longer timescales of Retransmission Time Out (RTO). It provides two mechanisms for packet loss detection: Fast Retransmit and timeout.

TCP interprets receipt of three duplicate ACKs as an indication of a packet loss. It retransmits the lost packet immediately upon the receipt of the third duplicate ACK. This mechanism is called Fast Retransmit; it detects a packet loss and reacts to it on the order of a flow's RTT. Another mechanism to detect a packet loss is the timeout mechanism. TCP sender starts a retransmission timer when it sends a packet. In case it receives less than three duplicate ACKs and the timer expires, the sender retransmits the packet. The initial RTO value is set to three seconds [3]. To keep balance between waiting time and spurious retransmission there exists a lower bound of one second for RTO value [3], [19].

The main reasons for the response-time performance degradations experienced by short TCP flows is their poor knowledge about the actual level of congestion in the network. Indeed, given that such flows only have a few packets to send, in case a packet gets lost in the network, they have no other option but to wait for the RTO to expire. In other words, they are unable to resend the packet immediately after one RTT, because the three duplicate ACKs may never return; simply because the corresponding data packets were never sent by the sender. Given that RTTs are typically of the order of 10's to 100 's of msec, each such event degrades the response time for approximately one to two orders of magnitude.

While the above effect has mainly been explored in the context of web traffic [20], [21], the same problem holds for interactive applications [5]. In such scenarios, a client typically sends a small burst of data, and then waits for a longer period of time (e.g., a few seconds) before sending the next burst. One additional issue with interactive scenarios is that even if an application manages to develop large congestion windows during burst periods, it cannot "freeze" the window during times when no data is coming from the application, and reuse it afterwards. Indeed, because the network conditions may change quickly, TCP endpoints are required to reduce their congestion windows during periods of data starvation [8].

\section{B. Implications}

All these facts give incentive for clients of interactive applications for misbehavior. The logic is simple: if interactive flows experience performance degradations relative to long TCP flows, then why not upgrading interactive to long flows? Clients can simply send packets into the network even when they have no data to send at a TCP fair rate, thus improving their performance in moments when they do have data to send. Figure 1 depicts this approach. Whenever data packets are available, they are immediately sent (hence, strict priority); in 


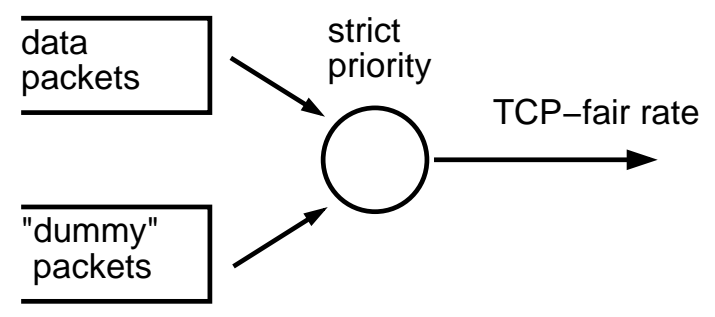

Fig. 1. Padding misbehavior: Upgrading mice to elephants.

times of application-level data starvation, "dummy" packets are sent into the network. A client can modify the kernel TCP implementation to achieve this functionality.

Incentives for clients to apply this approach are manifold. First, by adopting fully-backlogged approach, clients avoid losing memory in moments of data starvation [8]. Larger congestion windows can help "jumpstart" an actual data burst arriving from the application. Second, "dummy" packets following data packets may significantly increase the probability that a potential packet loss will be detected via the tripleduplicate ACK mechanism rather than the RTO. Finally, clients can freely apply this approach, without any fear of "getting caught." This is because both network- and endpointbased schemes designed to check for TCP-fairness compliance (e.g., [12]-[14]) would detect no violations.

Unfortunately, even though no law is officially violated with the above approach, its wide-spread adoption has a strong potential to seriously jeopardize the overall Internet performance. When only a single interactive flow is upgraded to a fully-backlogged flow, there is only a negligible or no increase in overall packet loss ratio [22]. Thus, the user sees an immediate improvement in its response time. However, it will aggravate the congestion and greatly increase the overall packet loss when everyone adopts this approach. Indeed, if interactive clients would start taking their bandwidth fairshare, the network would soon become highly congested. The packet-based Internet as we know it would soon become a "circuit-based" network; given the large number of short and interactive flows [20], [23], the bandwidth "dedicated" to each "TCP-friendly circuit" would soon converge to zero [22]. Still, our research indicates that even in such scenarios, misbehaving clients would outperform the behaving ones. Thus, the dangerous incentive remains.

Finally, implementing the approach of Figure 1 is not particularly challenging. Client-side only implementations, both at the TCP and the application levels are straight forward. Such designs could improve the times required to "push" packets to servers, a feature of particular interest to online gaming players. (Many online games require reliable transport, and hence use TCP ports [6]). While slightly more challenging, provoking servers to send at TCP-friendly rates is not impossible. One example is a recently proposed mobile TCP code method [12]. It enables clients to deploy a desired TCP version at servers. Given that it only checks for TCP friendliness, the approach of Figure 1 would not be qualified as a violation.

\section{PAdDing-Induced Response-Time Gains: MODELING AND Simulation}

Here, we quantify the gain a misbehaving client is able to achieve by applying the fully-backlogged approach. The key performance metric is the response time, defined as the time that elapses between sending a data packet into the network and receiving a corresponding acknowledgement. To establish a baseline for comparisons, we initially model the performance of pure interactive flows. Next, we model the response times achievable by fully-backlogged flows, assuming both random and correlated packet losses in the network. Finally, we verify our modeling results via simulation.

\section{A. Modeling Response Times of Application-Limited TCP Flows}

Interactive applications transmit data in cycles. The traffic generated by these applications can be characterized by two parameters: the data burst size, and the inter-burst time. Similar approach has been used to model telnet and gaming traffic in [24] and [25]. Both parameters are dependent on human behavior and activities, such as the user think times or the typing speed. The burst sizes are typically small, and they easily fit into a single packet [24], [25]. The inter-burst arrival times differ from application to application. They are typically modeled by the exponential distribution, with the mean of several hundreds of milliseconds (e.g., for gaming [24]) to several seconds (e.g., telnet [25]). In any case, as long as the inter-packet arrival times are longer than one third of the $R T O$, a potential packet loss will not trigger the triple-duplicate ACK mechanism, but will rather be detected via the RTO.

Thus, assuming single-packet-long data bursts and the RTObased packet-loss detection, we proceed as follows. Denote by $p$ the packet loss probability. We assume independent packet losses [16], [18]. While packet losses in a Droptail queue might be correlated within a RTT round, packet losses are independent among different rounds [18]. Given that interactive applications typically send only a single packet in a round, the independent packet loss assumption is reasonable for both RED and Droptail queues. Let $P_{h}(i)$ be the probability that a packet experiences exactly $i$ failure transmission attempts, followed by one successful try. Then,

$$
P_{h}(i)=p^{i}(1-p) .
$$

After the timeout expires, the client doubles the current value of RTO; thus, after $i$ consecutive packet losses, the RTO value is set to $2^{i} R T O$. Denote by $L(i)$ the corresponding latency experienced by the client after $i$ failure transmission attempts. $L(i)$ can be expressed as

$$
\begin{aligned}
L(i) & =\sum_{k=0}^{i-1} 2^{k} R T O+R T T \\
& =\left(2^{i}-1\right) R T O+R T T .
\end{aligned}
$$

Thus, for $p<0.5$, the expected value of the response-time latency becomes 


$$
\begin{aligned}
E[L] & =\sum_{i=0}^{\infty} P_{h}(i) L(i) \\
& =\left(\frac{p}{1-2 p}\right) R T O+R T T .
\end{aligned}
$$

\section{B. Modeling Response Times of Fully-Backlogged TCP Flows}

Here, we model the response times of fully-backlogged network-limited TCP flows. By establishing this result, we become capable of understanding gains that a misbehaving client can achieve by applying the fully-backlogged approach. We exploit the sophisticated modeling results of [15], [18], and further extend them to obtain the desired response-time characteristics. In our analysis, we consider both correlated and random packet losses, typical for DropTail and RED routers, respectively.

1) Correlated Packet Losses: Padhye et al. [18] develop the well-known TCP throughput model for fully-backlogged TCP flows, which we exploit to obtain the response-time characteristic. We use the same notation and preserve all relevant assumptions of [18]. From our perspective, the most important is the correlated packet loss assumption. It says that if a packet is lost, so are all the following packets within the same RTT round. Indeed, when the bottleneck router applies DropTail queuing, this is likely the case.

Denote by $b$ the average number of packets acknowledged by each ACK. Denote by $w$ the TCP congestion window size in packets, and by $E[w]$ its expected value. Then, according to $[18], E[w]$ becomes

$$
E[w]=\frac{2+b}{3 b}+\sqrt{\frac{8(1-p)}{3 b p}+\left(\frac{2+b}{3 b}\right)^{2}} .
$$

Next, for a given $w$, denote by $\hat{Q}(w)$ the probability that a loss is indicated via a timeout. According to [18],

$\hat{Q}(w)=\min \left(1, \frac{\left(1-(1-p)^{3}\right)\left(1+(1-p)^{3}\left(1-(1-p)^{(w-3)}\right)\right)}{1-(1-p)^{w}}\right)$

$Q$, the probability that a loss indication is a timeout is,

$$
Q=\sum_{w=1}^{\infty} \hat{Q}(w) P(W=w) \approx \hat{Q}(E[w]) .
$$

Consequently, the probability that the sender detects a packet loss via triple duplicate ACKs is given by $1-Q$.

2) Random Packet Losses: Brosh et al. [15] show that the above model underestimates the fast retransmit and fast recovery TCP features when routers deploy RED. Because in such scenarios packet losses are random, rather than correlated, the loss recovery probability increases and the subsequent loss recovery latency decreases. Thus, by adopting the Bernoulli loss model, the assumption is that each packet in a round is dropped with probability $p$, independently of other packets. Let $B(w, k)=\left(\begin{array}{l}w \\ k\end{array}\right) p^{w-k}(1-p)^{k}$. Then, according to [15], for

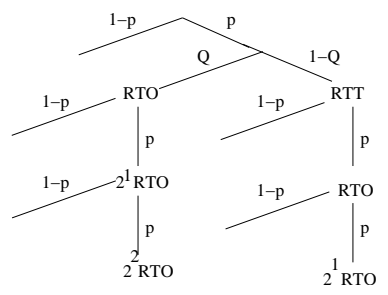

Fig. 2. Decision Tree

a given $w$, and for $w>3$, the probability that a loss indication is a timeout is given by

$$
\hat{Q}(w) \leq \frac{\sum_{k=0}^{2} B(w, k)\left(1+(1-p)^{k}\left(-1+(2-p)^{w}\right)\right)}{1-(1-p)^{w}}
$$

Next, assuming a uniform distribution for the TCP congestion window $W$, which is the instantaneous congestion window size based on TCP's congestion control algorithm, on the discrete interval $\left[0, w_{\max }\right]$; according to [15], the probability that a loss indication is a timeout becomes

$$
\begin{aligned}
Q & =\sum_{w=1}^{w_{\max }} \hat{Q}(w) P[W=w] \\
& \approx \min \left(1, \frac{1}{w_{\max }}\left(6+96 p-32 p^{2}+o\left(p^{3}\right)\right)\right) .
\end{aligned}
$$

Again, the probability that the sender detects a packet loss via triple duplicate ACKs is given by $1-Q$.

3) Response Times: Finally, we compute the response times for both of the above scenarios. One important issue here is that TCP always evokes an RTO if a retransmitted packet is lost again [17]. All versions of TCP, including NewReno and SACK, cannot recover from a retransmission loss without a retransmission timeout. Figure 2 depicts this effect. Once a packet is lost (with probability $p$ ), the triple-duplicate ACK mechanism will be invoked with probability $1-Q$. However, if the packet is lost more than once, the RTO is inevitable. While it may appear that computing $Q$ is not that essential (given that it appears only once in the decision tree), this is not the case. Given that the Q branch is close to the root of the tree, it does impact the response times in a non-trivial way, as we demonstrate below.

For a fully-backlogged TCP connection, denote by $L^{\prime}(i)$ the latency experienced by the client after exactly $i$ failure transmission attempts of a packet, followed by a successful transmission. Using the decision tree of Figure 2, we derive $L^{\prime}(i)$ as

$$
L^{\prime}(i)= \begin{cases}R T T & \text { for } i=0 \\ R T T+Q\left(2^{i}-1\right) R T O+ & \\ (1-Q)\left(R T T+\left(2^{i-1}-1\right) R T O\right) & \text { for } i \geq 1\end{cases}
$$

Consequently, the expected value of the response-time latency becomes 


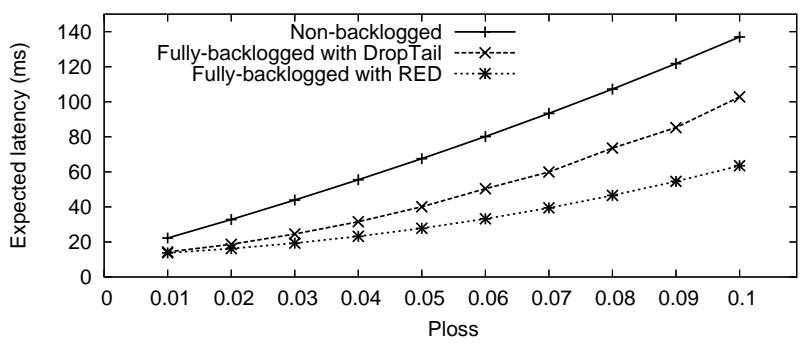

Fig. 3. Modeling: Expected latency as a function of packet loss prob.

$$
\begin{aligned}
E\left[L^{\prime}\right] & =\sum_{i=0}^{\infty} P_{h}(i) L^{\prime}(i) \\
& =Q\left(\frac{1-p}{1-2 p}-1\right) R T O+R T T \\
& +p(1-Q)\left(\left(\frac{1-p}{1-2 p}-1\right) R T O+R T T\right) .
\end{aligned}
$$

Finally, we define the response time gain, $G$, as the ratio between the expected response times for an interactive and a fully-backlogged TCP, $G=E[L] / E\left[L^{\prime}\right]$.

\section{Modeling Results}

Figure 3 depicts the expected latency as a function of the packet loss probability for application-limited as well as fully-backlogged flows (both for random and correlated packet losses). Naturally, in all scenarios, the expected latency increases as the packet loss probability increases. However, the key point is that for a given packet loss rate, the fullybacklogged flows always outperform interactive ones. In other words, clients promoting their flows from mice to elephants always experience better performance than pure interactive flows. Unfortunately, this means that the incentive for conducting the misbehavior is always present.

Figure 3 further shows that the padding misbehavior pays off better for RED-based bottlenecks. Because packet losses are random, avoiding RTOs is more likely in such scenarios. In particular, if a packet is lost, the probability that the following packets from the same RTT round will make it to the destination (and the corresponding ACKs back to the source) is not small. As a result, the triple duplicate ACK probability $(1-Q)$ is larger for random packet losses than for correlated ones. Figure 3 demonstrates that there still exists gain of fully-backlogged flows with DropTail over the pure interactive scenario; this is despite the correlated packet loss assumption (if a packet is lost in a round - then all packets that follow in the same round are dropped). If at least three packets from a RTT round make it to the destination before the concerned packet is lost, they may still trigger the tripleduplicate ACK mechanism in the following RTT round (see reference [18] for details).

Figure 4 depicts the response-time gains achievable by the padding misbehavior as a function of the packet loss ratio. Such a measure is of particular importance for misbehaving clients trying to maximize their performance gains. All curves in Figure 4 show a similar shape. Initially, the gain is relatively small for very small packet loss ratios. Indeed, even if packet losses are detected via the RTO, such events are rare, and thus the impact on the expected latency is negligible. However, as the packet loss ratio increases, so does the gain. Interactive flows suffer more and more, while fully backlogged flows manage to improve their performance by relying on the triple-duplicate ACK mechanism. Finally, the gain starts to decrease as the packet loss ratio keeps increasing. In such environments, the TCP congestion window starts reducing, Q starts converging to 1 , and padding is not as beneficial any more.

Figure 4 further shows that the gain is a function of RTT; the higher the RTT value, the smaller the RTO/RTT ratio, and the smaller the gain. Also, as RTT increases, the maximum gains are achieved for larger packet loss ratios. Indeed, as the $R T O / R T T$ ratio decreases, it must be compensated by its factor $((1-p) /(1-2 p)-1)$ (Equations (3), (10)) to keep a balance, meaning that $p$ increases. Finally, for the reasons explained above, RED's gain is larger than DropTail's.

\section{Simulation}

To verify our modeling results, we have conducted extensive simulation experiments, which are described here. The topology consists of a client and a server pool that are interconnected by a pair of routers and a bottleneck link. The effective round trip time fluctuates in the range from 10 to $100 \mathrm{~ms}$; likewise, we vary the bottleneck link capacity from 1.5 to $10 \mathrm{Mbps}$. By generating the background cross traffic of appropriate intensity, we control the packet loss ratio at the bottleneck. We use ns-2's TCP/FullTcpAgent. For each data sample, we run the simulation for a thousand seconds repeatedly and report averages ${ }^{1}$.

For interactive traffic, we open a telnet connection. The telnet client generates packets using an exponential distribution with average inter-arrival time of 1 second. For fullybacklogged TCP connections, we open FTP connections between a pair of nodes, one each from the server and the client pool. To accurately emulate an interactive connection converted to a fully-backlogged connection, we mark packets randomly using the same exponential distribution as in the telnet scenario. For the analysis of the simulation results, we consider the statistics for those marked packets only.

Figure 5(a) plots the simulation results for the gain ratio (the $y$-axis in the figure) as a function of the packet loss rate (the $\mathrm{x}$-axis in the figure) for RED and DropTail queues. In this particular scenario, we set the bottleneck bandwidth to $5 \mathrm{Mbps}$, and the round-trip propagation delay is $12 \mathrm{~ms}$. The bottleneck router buffer is $40 \mathrm{kB}$; in the RED case, we use the default $n s$ 2 RED parameters. We control the packet loss ratio by varying the intensity of the cross-traffic. The background traffic is composed of http, ftp, and low-rate udp traffic. In addition, we generate a pure interactive flow and an interactive flow converted to a fully-backlogged flow. For each of the flows, we $\log$ the experienced packet loss ratio and response-time. Then,

\footnotetext{
${ }^{1}$ The modified ns-2 code and simulation scripts are available at http://networks.cs.northwestern.edu/Intcp/code/
} 


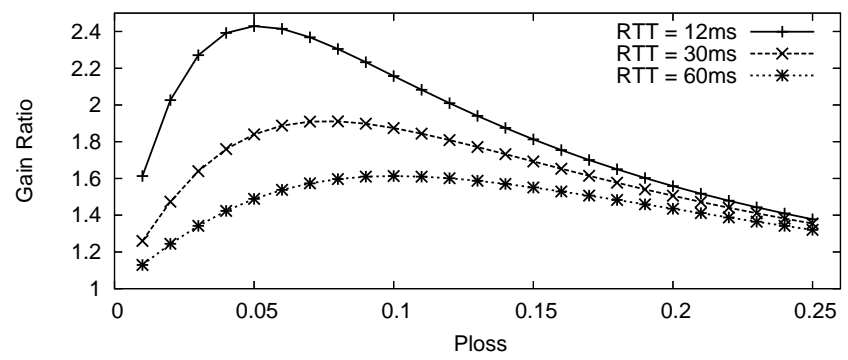

(a) RED

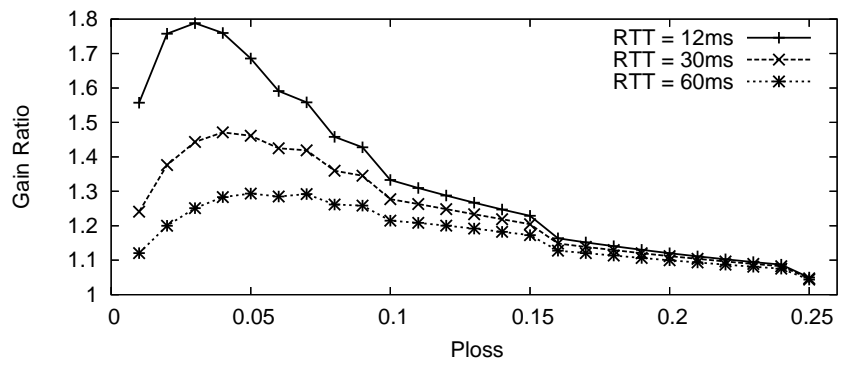

(b) DropTail

Fig. 4. Modeling: Gain ratio as a function of packet loss prob.

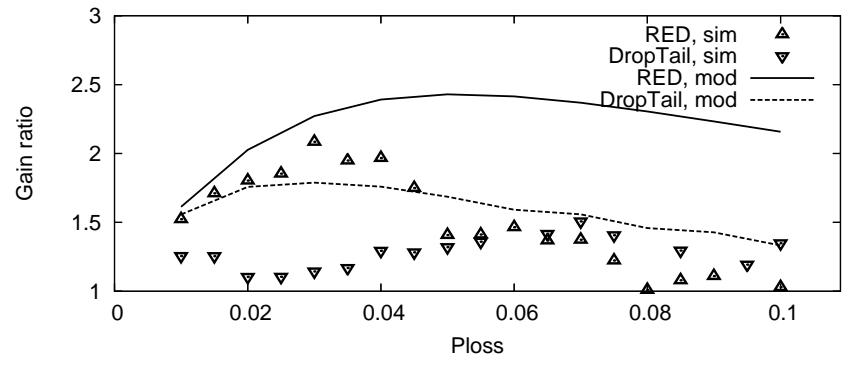

(a) congestion-related losses

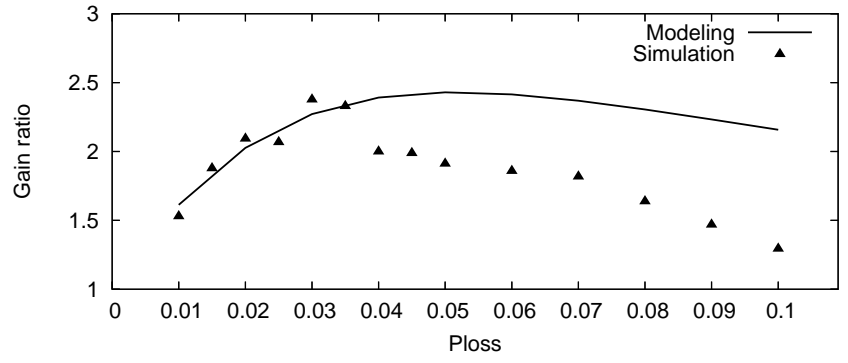

(b) artificial losses with RED queue

Fig. 5. Simulation: Gain ratio as a function of packet loss prob.

we run the experiment multiple times and aggregate the results to calculate the gain ratio. Unless otherwise indicated (e.g., Figure 8), this is the default simulation scenario. The shape of both curves in the figure is as predicted by modeling. Likewise, simulations confirm that gains are larger in the case of RED than with DropTail. The figure shows a discrepancy between the model and the simulations. This is due to varying queuing delay in simulations, and because the effective RTT increases with the packet loss ratio (congestion in the network), which is not captured in the model.

In order to understand why the match between modeling and simulations shown in Figure 5(a) is so poor, we proceed as follows. By applying the $n s-2$ 's artificial random packet loss module, we manage to effectively control the packet loss ratio while keeping the RTT value relatively constant. We apply RED queue at the routers for this experiment. Given that packet loss is introduced artificially, and there is no congestion in the network, both RED and DropTail yield similar results. Figure 5(b) shows the results. For packet loss ratios of up to $3.5 \%$, the model and simulations match well. However, for larger packet loss ratios, the modeling leads to over-estimation of the gain ratio. This is because we assumed that the initial RTO is set to minRTO of 1 second [3]. However, when the packet loss ratio is high, this is not necessarily the case. For example, due to multiple packet losses in a single RTT round, a future packet may "inherit" a longer initial RTO, an effect that is not captured in our modeling. Still, the gain in both scenarios remains in favor of fully-backlogged flows. Despite the mismatch between the modeling and simulations, the gain ratio is always greater than one both for RED and Droptail queues. The important implication is that greedy users always have the incentive to adopt the fully-backlogged approach to improve their response times.

In order to understand the impact on overall network performance when a user adopts the fully-backlogged approach we conduct an experiment and present our observation in Figure 6. The experiment is done using a dumbbell topology with access link capacity of $100 \mathrm{Mbps}$ and bottleneck capacity of $10 \mathrm{Mbps}$. Each link in the topology has a delay of $2 \mathrm{~ms}$. Figure 6 shows the overall loss rate on the Y-axis and number of fully-backlogged flows in the system on the $\mathrm{X}$-axis. The gap between the solid line and the dotted line depicts the increase in packet loss rate when a user adopts the fully-backlogged approach for a given number of background flows on $\mathrm{X}$-axis.

Figure 6 clearly shows that there is no considerable increase in packet loss rate for any given background setup when a user upgrades itself to fully-backlogged connection. Given this observation and the fact that an interactive connection always improves its response times by adopting fully-backlogged approach, any greedy user would always be tempted to upgrade itself to fully- backlogged connection, even after knowing that if everyone starts doing the same the overall Internet performance will be severely degraded. This is because there is no central authority that monitors or controls user behavior in the Internet, and a user has no way to know what others are doing. Thus upgrading itself is the best strategy for a user, and any greedy rational user will do it. 


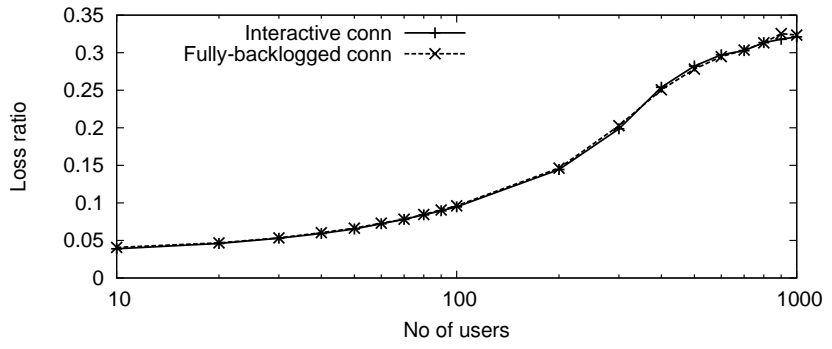

Fig. 6. Loss rate as a function of number of users

\section{Sustainable Countermeasures}

In this section, we explore ways to enhance the performance of interactive applications without applying the fullybacklogged approach. In other words, the challenge is to make sustainable changes, which if applied globally, would $(i)$ solve the problem, yet (ii) without compromising the idea of statistical multiplexing. Our primary goal is to increase the performance of legitimate users to a level which will demotivate misbehaving clients from converting their interactive flows into fully-backlogged TCP connections.

\section{A. Approach I: Differentiated minRTO}

We initially focus on the RTO parameter. Selection of the timeout value requires a balance among two extremes: if set too low, spurious retransmissions will occur when packets are incorrectly assumed lost when in fact the data or ACKs are merely delayed. Similarly, if set too high, flows will wait unnecessarily long to infer and recover from congestion. Allman and Paxson [19] experimentally showed that TCP achieves near-maximal throughput in the Internet if there exists a lower bound for RTO of one second. The study found that all flows should have a time-out value of at least 1 second in order to ensure that congestion is cleared, thereby achieving the best performance.

One approach to reducing the performance degradations experienced by application-limited flows is reducing the minRTO parameter exclusively for such flows. In particular, we explore an approach in which a TCP sender is allowed to use a lower value for minRTO, (e.g., minRTO'), when its used congestion window size is less than a fraction of the current congestion window size (we quantify the minRTO' and fraction parameters below). While it is arguable whether such an approach can cause a congestion collapse, one argument in its behalf is that interactive applications represent only a small fraction of the byte-level Internet traffic. Moreover, the ultimate protector from congestion collapse is the RTO backoff mechanism [26]. Thus, having a few spurious retransmissions will not degrade the network performance by a perceptible amount. Moreover, in the context of the congestion collapse problem, this approach can only be better than the fullybacklogged one. However, the results we present below make such a discussion obsolete.

\section{B. Approach II: Short-term padding with dummy packets}

In this approach, the goal is to improve the performance of interactive applications by increasing the probability that a packet loss is detected via the fast retransmit mechanism; yet, without applying the "brute-force" fully-backlogged approach. In particular, this could be achieved by appending the application data packet with three "tiny" (e.g., 20 bytes each) "dummy" packets. Indeed, RFC 3390 [27] enables setting TCP's initial congestion window size to 4 packets when TCP starts a new connection or restarts a connection after a long idle period. Thus, the three additional tiny dummy packets should help the endpoints detect data packet losses via triple dummy-packet-initiated duplicate ACKs.

The unique characteristic of this approach is that in addition to being implementable at the TCP layer, it could be implemented at the application level as well. An application should make sure that it does not send packets back to back; otherwise, TCP will make a single 60-byte packet and send it to the network. At any rate, contrary to the approaches above and below, interactive applications could immediately deploy this approach without requiring any kernel-level TCP changes.

1) Modeling: Here, we derive the response-time formula for the short-term padding approach. Assume a general scenario in which the minimum congestion window size parameter is $m$, such that $m-1$ packets are appended to a data packet. A timeout is invoked if two or fewer dummy packets reach the receiver; more precisely, if the TCP sender gets back two or less duplicate ACKs. Thus, the probability that the loss indication for a data packet is a timeout is given by

$$
Q(m)=\sum_{i=0}^{2}\left(\begin{array}{c}
m-1 \\
i
\end{array}\right) p^{(m-1-i)}(1-p)^{i} .
$$

Again, the probability that a data packet loss is detected by the triple duplicate ACK mechanism is 1-Q. Also, as discussed above, in case a retransmitted packet is lost again, it evokes an RTO. Thus, by applying the same approach as in Section III-B, the expected latency becomes

$$
\begin{aligned}
E\left[L^{\prime}\right] & =Q\left(\frac{1-p}{1-2 p}-1\right) R T O+R T T \\
& +p(1-Q)\left(\left(\frac{1-p}{1-2 p}-1\right) R T O+R T T\right) .
\end{aligned}
$$

Strictly speaking, Equation (12) applies only to the random loss scenario. Indeed, under the assumption that if a packet is lost, so are all the packets that follow in the same round, the proposed approach is ineffective. However, our simulations indicate that the above correlated packet loss assumption is "too strong," and that when a data packet is lost, the corresponding follow-up packets are not always dropped in DropTail routers. Hence, the proposed approach improves the performance even in such scenarios, as we show below.

\section{Approach III: A Diversity Approach}

In this approach, we modify a TCP sender to send a packet $k$ times; $k$ is a small integer, $k>1$. TCP sends $k$ copies of a packet without violating TCP's congestion control mechanism. The key idea behind this approach is that the probability that at least one of the $k$ copies of a packet will make it to the receiver 
is high. However, if all $k$ packets are lost, TCP undergoes retransmission timeout and cuts down the congestion window to one. Hence, in the following retransmission rounds, it retransmits the packet only once.

Formally, when a TCP sender transmits only a single packet and it cannot send any more packets because it does not have any data to send, this approach allows the TCP sender to transmit a duplicate copy back to back. Of course, this only applies under the assumption that the current congestion window permits the TCP sender to transmit more than one packet.

This is a TCP-only approach; it cannot be deployed at the application layer. For example, if two copies of a packet are sent from the application to the TCP layer, TCP will treat them as two different packets. Thus, if the first packet is lost and the second one makes it to the receiver, the second packet will only be buffered at the TCP layer; it will be "pushed" to the application layer only after the first packet is successfully retransmitted - which in this scenario happens after one RTO in the best case.

Note that TCP uses the lack of an acknowledgement as an implicit signal of packet loss, and reacts by cutting down its congestion window. However, in this approach, even if one of the packets is lost, TCP sender gets acknowledgement from the receiver. In order to keep TCP's congestion mechanism function correctly, the sender maintains extra information, whenever it sends a duplicate copy, it expects a duplicate acknowledgement from the receiver. If it does not receive the duplicate acknowledgement, it cuts down the congestion window. Moreover, delayed acknowledgement should be disabled at the receiver.

1) Modeling: Here, we derive the response-time formula for the diversity approach. Denote by $k$ the number of copies of a packet a TCP sender generates. Then, the probability that at least one copy of a packet is transmitted successfully exactly after $i$ failure rounds becomes

$$
P_{h}(i)= \begin{cases}1-p^{k} & \text { for } i=0 \\ p^{k+i-1}(1-p) & \text { for } i \geq 1\end{cases}
$$

In particular, the probability that at least one of the $k$ packets in the first round successfully reaches the destination is given by $1-p^{k}$. For $i \geq 1, P_{h}(i)$ is given by the product of two probabilities: $(i)$ the probability that all $k$ copies are lost in the first round, and ( $i i)$ the probability that the single packet copy is lost in all subsequent $i-1$ rounds, followed by a successful transmission. Then, following the approach of Equation (3), it could be shown that for $p<0.5$, the expected response-time latency becomes

$$
E\left[L^{\prime}\right]=p^{k-1} R T O\left(\frac{(1-p)}{(1-2 p)}-1\right)+R T T .
$$

Similarly to the above scenario, our modeling approach here strictly applies only to random packet losses. Still, simulations below indicate that the approach is viable in DropTail scenarios as well.

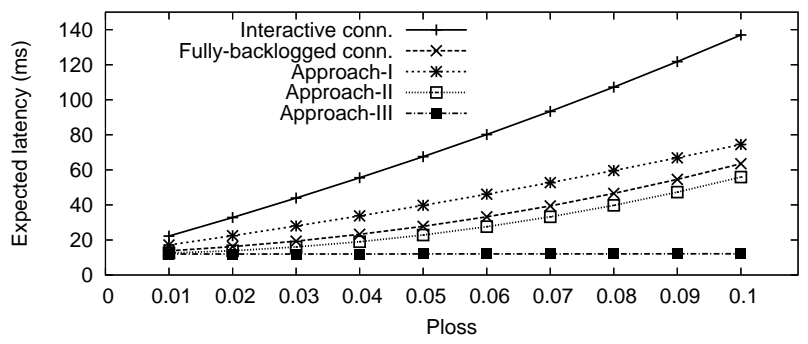

Fig. 7. Modeling: Expected latency as a function of packet loss prob.

\section{Evaluation}

Here, we evaluate the effectiveness of the three approaches. For Approach I, we set the minRTO' to $500 \mathrm{~ms}$, and the fraction parameter to $1 / 4$. Given that the minimum congestion window is four packets [27], this enables a client to always re-send a packet after an RTO of $500 \mathrm{~ms}$. In Approach II, an application data packet is appended with three applicationlevel dummy packets, each of the size of 20 bytes. After TCP adds a 40-byte-long header, the dummy packet size in the network becomes 60 bytes. Finally, for Approach III, we set $k=2$; thus, each packet is repeated twice.

Figure 7 plots the expected latency, estimated by the analyses given above (Equation 12,14), as a function of the packet loss ratio for the three approaches. The key observation is that the short-term padding and diversity approaches outperform the fully-backlogged approach. In this way, two goals are achieved: $(i)$ The interactive-application clients no longer have incentives to generate fully-backlogged flows. Indeed, why convert to fully-backlogged when approaches-II and -III are better? (ii) Approaches-II and -III still preserve the idea of statistical multiplexing, as we demonstrate below.

Figure 7 also shows that despite the fact that we reduced the minRTO parameter by a half, the response time of Approach I is still higher than that of the fully-backlogged approach. Our evaluations (using both Equation (3) and simulations) indicate that reducing the minRTO parameter much more would help outperform the fully-backlogged approach. However, such an approach in essence converges to the Approach III (for $k=2$ ), and hence we refrain from showing it further.

1) Simulations: Figure 8 plots the simulation results for the above scenarios, both for RED and DropTail routers. In simulations, we control the packet loss by varying the intensity of the cross traffic. In addition, we generate one of the flows indicated in the figure: an interactive, a fully-backlogged, an Approach II, and an Approach III flow. Figure 8(a) (the RED case) confirms general trends shown previously in Figure 7: approaches-II and -III outperform the fully-backlogged scenario. Moreover, as explained above (in Section III-D), due to inheriting longer than minRTO initial timeouts, the fully-backlogged flow experiences additional response-time degradations for larger packet-loss ratios.

Figure 8(b) plots simulation results for the DropTail case. While correlated packet losses, characteristic for drop-tail queues, do affect the overall performance, the key finding remains unchanged: both approaches-II and -III have lower response times than the fully-backlogged approach. For exam- 


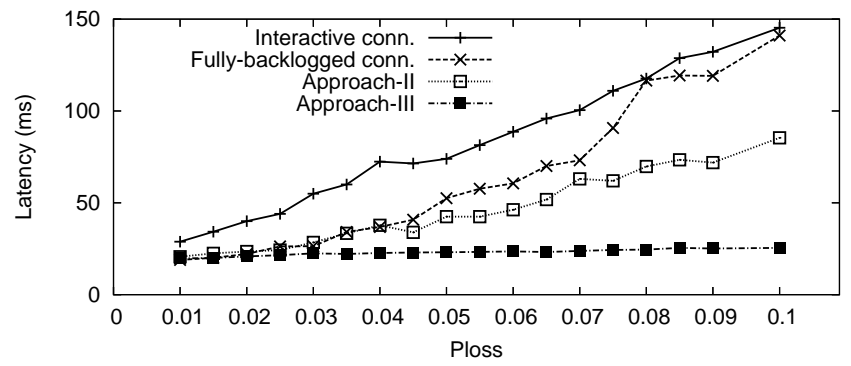

(a) RED

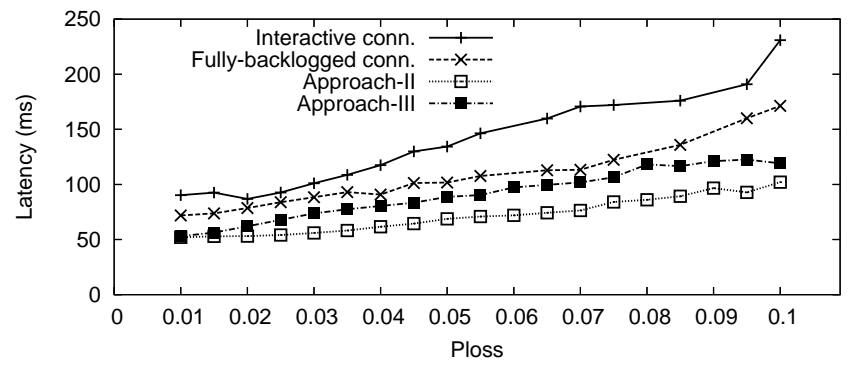

(b) DropTail

Fig. 8. Simulation: Latency as a function of loss rate

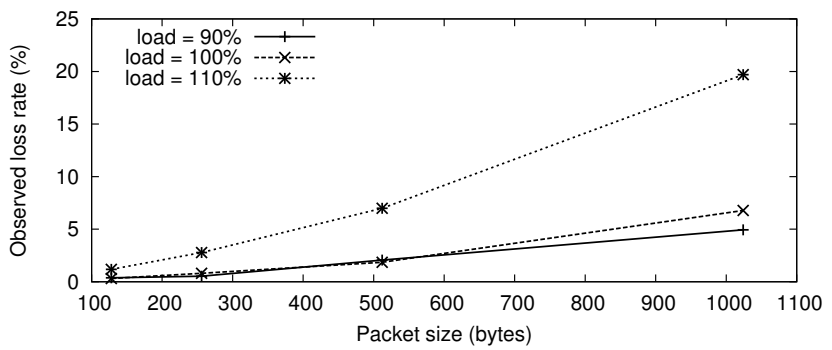

Fig. 10. Simulation: Observed loss rate as a function of segment size used by the flows.

ple, due to larger probability that both copies of a packet in Approach III will get dropped at the router, its performance is not as good as in the RED case. However, because the probability that both packets are lost concurrently does not equal one in reality, there still exists gain over the fullybacklogged approach. Also, contrary to the RED scenario, it is interesting that Approach II (padding) outperforms Approach III (diversity). Since padded dummy packets are smaller than data packets, the likelihood that they will get dropped at the byte-based drop-tail queue is smaller. To validate the hypothesis, we conduct an experiment where we generate multiple Telnet flows, and each of them uses a different segment size (128, 256, 512, and 1024 bytes). The bottleneck router uses a Droptail queue in the byte mode. We control the network load by generating pareto and http cross traffic of average segment size of 576 bytes. Figure 10 shows the impact of the packet size on the observed loss rate experienced by the flows. It clearly shows that flows with smaller segment sizes experience significantly lower loss rates relative to the flows with larger segment sizes.

2) Overhead and Sustainability: One final issue that we explore is overhead and sustainability. In essence, we explore scenarios in which a given approach is widely deployed, and evaluate $(i)$ the performance gains over the greedy fullybacklogged approach, and $(i i)$ performance reductions relative to the purely interactive, yet unsustainable, approach.

Figure 9 plots response times as a function of the number of flows in the network, when all clients apply a given approach indicated in the figure. Even for a moderate number of connections, the response times increase dramatically for the fully-backlogged approach. At the other extreme, the purely interactive approach can support more than 350 connections before the latency starts increasing. Unfortunately, as discussed above, this state is unstable in the sense that clients have incentives to improve their performance while still remaining TCP friendly. Finally, the figure shows that approaches-II and -III support a necessarily smaller number of connections relative to the interactive scenario. However, the key point is that both approaches provide (i) a sustainable solution that demotivates clients from moving the system into the fully-backlogged state; and (ii) a significantly "friendlier" environment relative to the fully-backlogged approach.

Figure 9 shows that in the case of Approach III, the latency starts increasing when the number of flows exceeds 175. Indeed, because clients send two copies of a packet by default, the "departure" point is approximately at one half of the number achievable by the purely interactive approach. Next, because the overhead for the Approach II is smaller ( $3 * 60$ bytes relative to 540 bytes-long data packets), it can support a larger number of flows without increasing response times (the "departure" point is around 250 flows).

Also, while the performance for Approach II (padding) is approximately identical in the RED and DropTail scenarios, this is not the case with the diversity approach. Indeed, Figure 9(a) shows that RED's random packet dropping has a brilliant effect on Approach III, given that latency increases moderately with the number of flows. Not only that the approach dramatically outperform the fully-backlogged approach, but it even outperforms the pure interactive approach when there are many flows in the system. By contrast, due to correlated packet losses, the latency slope is much steeper in the DropTail case.

\section{E. TCP Smart Framing}

Mellia et al. [28] propose a segmentation algorithm, TCP Smart Framing (TCP-SF) to reduce TCP's latency. The basic notion is to split data into smaller segments when TCP's congestion window is less than four maximum segment sizes (MSS). This helps TCP to recover from packet losses during the slow-start period. In essence, this is an attempt to ensure that there are always enough packets in the network to trigger the fast retransmit. As the congestion window grows, the algorithm increases the segment size. This technique has been shown to perform well in reducing timeout events during the slow-start phase when the end point has new data to send in 


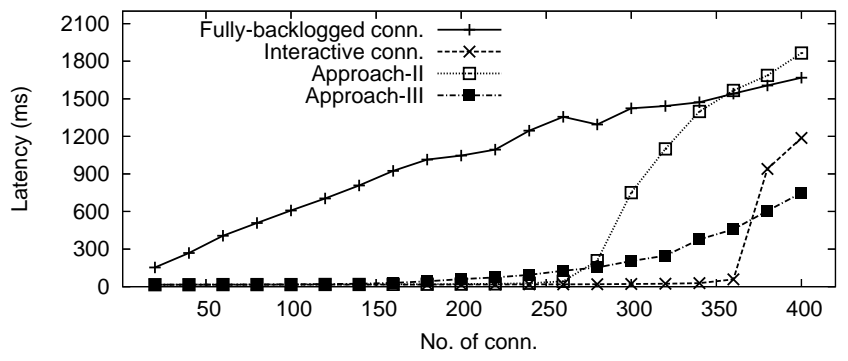

(a) RED

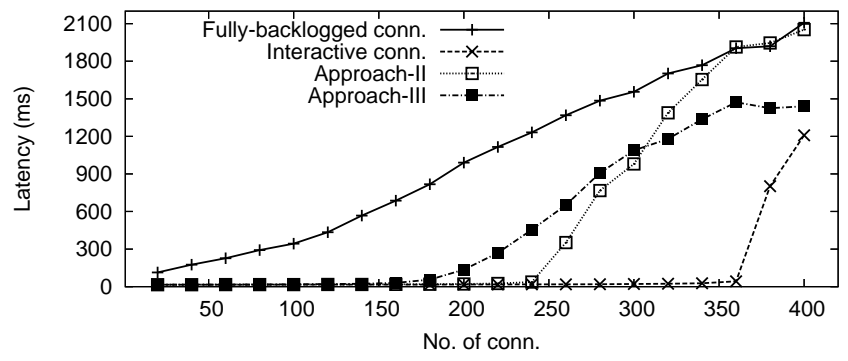

(b) DropTail

Fig. 9. Simulation: Number of flows vs latency: $\mathrm{C}=1.5 \mathrm{Mbps}$

response to an acknowledgement. Below, we explore if this approach can improve the performance of interactive flows.

It appears that TCP-SF approach will also enable interactive TCP senders to detect packet losses via fast retransmit without waiting for long retransmit timeouts. However, we show below that contrary to the common intuition, TCP-SF even degrades the response times for interactive applications. The key reason is that the probability of getting one of the multiple small segments lost in the network is even higher than when entire data is packed into a single packet. This approach works well for reducing timeout probability during the slow-start period of a backlogged TCP connection because the TCP sender always has new data to send upon a receipt of an acknowledgement. But, this does not hold true for an interactive TCP sender. Interactive applications often wait for the response message from its peer before sending any new data to the TCP layer. In such scenarios, if any of the last three segments are lost, then the only way to recover is to wait for the retransmit timer to expire. This exacerbates response time of interactive applications.

1) Modeling: Here, we analyze the behavior of the TCP$\mathrm{SF}$ approach using a state machine. Figure 11 describes the behavior of TCP-SF for a interactive connection. The initial state of the system is represented by $S$. When a loss occurs, TCP-SF can either enter fast retransmit (FR) or timeout (TO). This choice depends on the loss pattern. $F R$ can be entered only if the first segment is lost and the following three segments are successfully delivered. Denote by $p_{s}$ the smallsize segment loss probability. Then, the transition probability from state $S$ to $F R$ is equal to $p(S, F R)=p_{s}\left(1-p_{s}\right)^{3}$. If a loss pattern occurs so that $F R$ cannot be entered, the state machine moves from state $S$ to $T O$. The probability of this transition is $p(S, T O)=1-p(S, F R)-\left(1-p_{s}\right)^{4}$. The state machine can then leave state $T O$ and enter state $S$ if no further losses occur. Similarly, from state $F R$, it can either

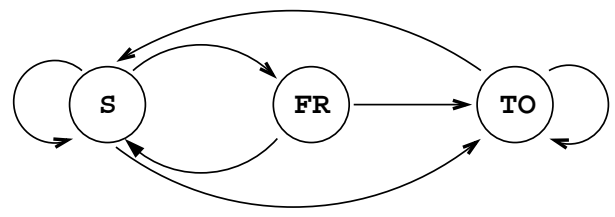

Fig. 11. State machine to analyze the behavior of TCP-SF enter $T O$ or go back to $S$ depending upon further loss events. The state machine of TCP for interactive connection consists of two states only; $S$ and $T O$. Denote by $p$ the normal-size packet loss probability; the probability of transition from state $S$ to $T O$ is equal to $p(S, T O)=p$.

The performance of the TCP-SF approach relative to the TCP approach highly depends upon small-size to normal-size $\left(p_{s} / p\right)$ packet loss ratio. In a packet-based queue, where $p_{s}=$ $p$, the TCP-SF approach will necessarily perform worse than TCP. Even with byte-based droptail queue, where the queue operates at the edge during periods of congestion, packet losses are inevitable when multiple small-size packets arrive in burst. This will often lead to multiple packet losses, and resulting in timeout. We evaluate these hypotheses below.

2) Simulations: Figure 12(a) shows the TCP-SF and TCP response time profiles for a byte-based droptail queue. The result is even worse for a packet-based queue (not shown). It clearly reveals that the response-time plot for TCP-SF is significantly worse than it is for TCP. This is for the same reason explained above. Figure 12(b) shows that there is little improvement when RED AQM is deployed at the network routers. This is because the average queue size remains below the actual queue limit due to the random early dropping mechanism, and the packet dropping probability reduces proportionally when the incoming packet size is smaller than the mean packet size [29]. However, both DropTail and RED queues are deployed in today's Internet, which makes TCP smart framing a nonviable solution for interactive TCP.

\section{RELATED WORK}

There has been substantial amount of work to improve the response time of short flows in the Internet. Many such approaches appear to address the response time degradation problem of interactive applications to some extent. In this section, we discuss such related work.

Several solutions based on the idea of service differentiation and preferential treatment to short flows in the network has been proposed. Guo and Matta [2] use different marking/dropping functions at the routers and a packet classifier at the network edge to distinguish between long- and shortlived TCP flows. In addition to requiring large changes to the existing network infrastructure, the solution appears to address the problem of short, but not the interactive flows 


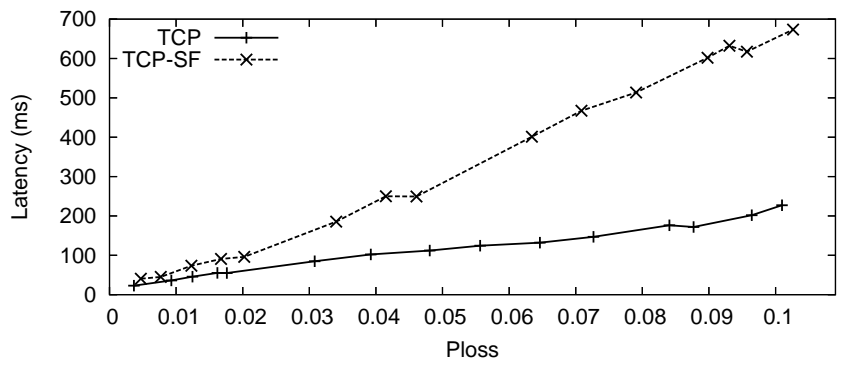

(a) DropTail

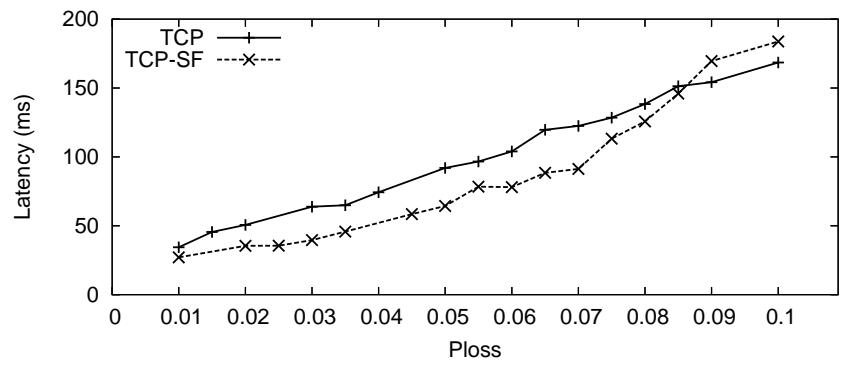

(b) RED

Fig. 12. Simulation: Evaluation of TCP-SF for interactive applications

because its packet/flow classification technique is based on packet counting, rather than the sending rate of the flow. It would classify a long-lived interactive connection, even if sending packets at a very low-rate, into the category of long flows, once the packet counter exceeds the threshold value. Noureddine and Tobagi [5] propose application- and TCP-level marking to give strict priority to interactive applications in the network. In addition to requiring per-user traffic policing at the network edge (tedious to deploy), the authors assume a widespread network support for multi-priority services in the Internet (to the best of our knowledge, not the case).

Le et al. [4] propose an AQM scheme which gives a strict priority to short flows, while it applies congestion control only to long flows. The key advantage over the above two schemes is that it requires no support from the endpoints; it distinguishes short from long flows by tracking the number of packets that have recently been seen from each flow at the router. In addition to provoking potential security and stability side effects (e.g., see [21]), the proposed scheme requires to be implemented in the network core; unfortunately, no strong incentives for such a deployment exist. Similarly, it has been shown that marking, instead of dropping, TCP control packets using Explicit Congestion Notification (ECN) could significantly improve the performance of short flows [21]. Unfortunately, ECN is not widely deployed in today's Internet.

Endpoint-based approaches have also been proposed. A large amount of Internet TCP trace analysis [30] shows that lowering TCP's duplicate acknowledgement threshold from three to two, would increase the fast retransmit opportunities by $65-70 \%$. However, it also increases the number of spurious retransmissions by a factor of three. Further analysis shows that introducing a small delay (around $20 \mathrm{~ms}$ ) at the receiver, before sending duplicate acknowledgement upon the advent of a sequence hole, can avoid most of such spurious retransmissions. Since this approach requires modification both at the sender and receiver side, the author discards this solution as being impractical for Internet wide deployment.

The Limited transmit approach [31] allows a TCP sender to transmit a previously unsent data upon arrival of the first two consecutive duplicate acknowledgements, provided, of course, that the receiver window allows the transmission of the segment and outstanding data remains less or equal to the congestion window size plus two segments. Assuming these two new segments and corresponding ACKs are not dropped, the sender can infer packet loss using standard Fast Retransmit threshold of three packets. To take advantage of this method, TCP sender should always have new data to transmit, which is not the case with interactive applications.

The Early Retransmit approach [32] addresses TCP's loss recovery mechanism for congestion windows smaller than four packets and when the TCP sender cannot send any new data either because of a limited receiver window or it does not have any new data to send. This approach enables TCP senders to retransmit the lost packet upon receiving congestion window minus one duplicate acknowledgements. However, the TCP sender should send at least two packets for this loss recovery mechanism to work.

To address the problem of low network observability by short flows, RFC 3390 [27] specifies an increase in the permitted upper bound for TCP's initial window from one or two segment(s) to between two and four segments. If at least one of the packets returns to the sender, the connection will not suffer the initial default 3 second-long timeout penalty [3]. Padmanabhan [33] describes a technique for integrated loss recovery and congestion window evolution by persistent and pipelined HTTP flows. Such techniques are shown to be capable of faster loss recovery and more responsive to network congestion than multiple TCP connections. The motivation behind integrated loss recovery is to reduce the number of timeouts for short flows. Yang and de Veciana [34] develop TCP/SAReno in which the AIMD parameters dynamically depend on the remaining file size, such that short flows become more aggressive. Finally, Savage et al. [35] and Anderson et $a l$. [36] have demonstrated that using history can be efficiently used to improve the performance of short flows.

Despite the fact that all of the above endpoint approaches enable protocol support for improving the performance of short or interactive flows, the key problem remains: the application-level data starvation can prevent clients from experiencing any benefits from the above designs. In particular, the burst periods of interactive flows are typically small enough to fit into a single packet [24], [25]. As a result, an increased congestion window, a more aggressive TCP, or a history-based approach cannot help. If a packet gets lost in the network, the sender must rely on the RTO mechanism 
before re-injecting the packet back into the network, thus experiencing significant performance degradations.

\section{CONCLUSIONS}

This paper points out the response time degradations suffered by interactive flows relative to fully-backlogged flows during periods of congestion; it demonstrates that interactive flows can improve their response times by converting themselves into fully-backlogged flows. Our first contribution lies in pointing out at an imminent and a serious implication of this problem: nothing stops clients of interactive applications to improve their response-time performance by generating traffic at a TCP-fair rate. The problem is imminent because the misbehavior is hard to detect, given that flows are TCP friendly. The problem is serious because it has the potential to jeopardize one of the core principles that today's Internet is built upon - statistical multiplexing. Second, we showed that interactive clients always have an incentive to send at a TCP-fair rate, because the corresponding response-time performance always outperforms the pure interactive approach. Moreover, we revealed that due to random packet losses, the gain is much larger for RED routers. Finally, we demonstrated that there exist simple, easy-to-deploy, and sustainable solutions that are capable of effectively demotivating clients from applying the greedy fully-backlogged approach. In particular, we showed that a diversity method, accompanied with RED routers in the network, performs remarkably well. Still, the short-term padding approach appears even more attractive; it could be implemented at the application layer, without requiring any $\mathrm{TCP}-$ level modifications.

\section{ACKNOWLEDGEMENTS}

We would like to thank Mark Allman and Sally Floyd for their insightful comments on this paper. We would also like to thank anonymous INFOCOM reviewers for their valuable feedback on an earlier version of this paper.

\section{REFERENCES}

[1] A. Mondal and A. Kuzmanovic, "When TCP friendliness becomes harmful," in Proceedings of IEEE INFOCOM '07, Anchorage, Alaska, May 2007.

[2] L. Guo and I. Matta, "The war between mice and elephants," in Proceedings of IEEE ICNP '01, Riverside, CA, Nov. 2001.

[3] V. Paxson and M. Allman, "Computing TCP's retransmission timer," Nov. 2000, Internet RFC 2988.

[4] L. Le, J. Aikat, K. Jeffay, and F. Smith, "Differential congestion notification: Taming the elephants,' in Proceedings of IEEE ICNP '04, Berlin, Germany, Oct. 2004.

[5] W. Noureddine and F. Tobagi, "Improving the performance of interactive TCP applications using service differentiation," in Proceedings of IEEE INFOCOM '02, New York, NY, June 2002.

[6] "Which ports are used by computer games?" http://www.u.arizona.edu/ trw/games/ports.htm.

[7] R. Fielding, J. Gettys, J. Mogul, H. Frystyk, L. Masinter, P. Leach, and T. Berners-Lee, "Hypertext transfer protocol - HTTP/1.1," June 1999, Internet RFC 2616.

[8] M. Handley, J. Padhye, and S. Floyd, "TCP congestion window validation," June 2000, Internet RFC 2861.

[9] P. Dinda, G. Memik, R. Dick, B. Lin, A. Mallik, A. Gupta, and S. Rossoff, "The User In Experimental Computer Systems Research," in Proceedings of the Workshop on Experimental Computer Science (ExpCS'07), June 2007.
[10] S. McCreary and K. Claffy, "Trends in wide area IP traffic patterns A view from ames Internet exchange," Proceedings of the 13th ITC Specialist Seminar on Internet Traffic Measurement and Modelling, 2000.

[11] T. Karagiannis, K. Papagiannaki, and M. Faloutsos, "BLINC: multilevel traffic classification in the dark," in Proceedings of ACM SIGCOMM '05, Philadelphia, PA, Aug. 2005.

[12] P. Patel, A. Whitaker, D. Wetherall, J. Lepreau, and T. Stack, "Upgrading transport protocols with untrusted mobile code," in Proceedings of ACM SOSP '03, Bolton Landing, NY, Oct. 2003.

[13] R. Mahajan, S. Floyd, and D. Wetherall, "Controlling high-bandwidth flows at the congested router,' in Proceedings of IEEE ICNP '01, Riverside, CA, Nov. 2001.

[14] A. Kuzmanovic and E. Knightly, "A performance vs. trust perspective in the design of end-point congestion control protocols," in Proceedings of IEEE ICNP '04, Berlin, Germany, Oct. 2004.

[15] E. Brosh, G. Lubetzky-Sharon, and Y. Shavitt, "Spatial-temporal analysis of passive TCP measurements," in Proceedings of IEEE INFOCOM '05, Miami, FL, Mar. 2005.

[16] N. Cardwell, S. Savage, and T. Anderson, "Modeling TCP latency," in Proceedings of IEEE INFOCOM 'O0, Tel Aviv, Israel, Mar. 2000.

[17] B. Kim and J. Lee, "Retransmission loss recovery by duplicate acknowledgement counting," IEEE Communications Letters, vol. 8, no. 1, Jan. 2004.

[18] J. Padhye, V. Firoiu, D. Towsley, and J. Kurose, "Modeling TCP Reno performance: A simple model and its empirical validation," IEEE/ACM Transactions on Networking, vol. 8, no. 2, pp. 133-145, Apr. 2000.

[19] M. Allman and V. Paxson, "On estimating end-to-end network path properties," in Proceedings of ACM SIGCOMM '99, Vancouver, British Columbia, Sept. 1999.

[20] L. Le, J. Aikat, K. Jeffay, and F. Smith, "The effects of active queue management on Web performance," in Proceedings of ACM SIGCOMM '03, Karlsruhe, Germany, Aug. 2003.

[21] A. Kuzmanovic, "The power of explicit congestion notification," in Proceedings of ACM SIGCOMM '05, Philadelphia, PA, Aug. 2005.

[22] R. Morris, "TCP behavior with many flows," in Proceedings of IEEE ICNP '97, Atlanta, GA, Oct. 1997.

[23] F. Smith, F. Campos, K. Jeffay, and D. Ott, "What TCP/IP protocol headers can tell us about the Web," in Proceedings of ACM SIGMETRICS '01, Cambridge, MA, June 2001.

[24] J. Farber, "Network game traffic modeling," in Proceedings of NetGames '02, Braunschweig, Germany, Apr. 2002.

[25] P. Danzig and S. Jamin, "tcplib: A library of internetwork traffic characteristics," USC Technical Report, Computer Science Department, 1991, Report CS-SYS-91-01.

[26] V. Jacobson, "Congestion avoidance and control," in Proceedings of ACM SIGCOMM'88, Stanford, CA, Aug. 1988, pp. 314-329.

[27] M. Allman, S. Floyd, and C. Partridge, "Increasing TCP's initial window," Oct. 2002, Internet RFC 3390.

[28] M. Mellia, M. Meo, and C. Casetti, "TCP smart framing: a segmentation algorithm to reduce TCP latency," IEEE/ACM Transactions on Networking, vol. 13, no. 2, pp. 316-329, 2005.

[29] W. Eddy and M. Allman, "A comparison of RED's byte and packet modes," Computer Networks, vol. 42, no. 2, June 2003.

[30] V. Paxson, "End-to-end Internet packet dynamics," IEEE/ACM Transactions on Networking, vol. 7, no. 3, pp. 277-292, June 1999.

[31] M. Allman, H. Balakrishnan, and S. Floyd, "Enhancing TCP's loss recovery using limited retransmit," Jan. 2001, Internet RFC 3042.

[32] M. Allman, K. Avrachenkov, U. Ayesta, and J. Blanton, "Early Retransmit for TCP and STCP," Nov. 2006, IETF Internet Draft.

[33] V. Padmanabhan, "Addressing the challenges of web data transport," PhD Dissertation, University of California, Berkeley, 1998.

[34] S. Yang and G. de Veciana, "Size-based adaptive bandwidth allocation: Optimizing the average QoS for elastic flows," in Proceedings of IEEE INFOCOM '02, New York, NY, June 2002.

[35] S. Savage, N. Cardwell, and T. Anderson, "The case for informed transport protocols," in Proceedings of HotOS '99, Rio Rico, Arizona, Mar. 1999.

[36] T. Anderson, A. Collins, A. Krishnamurthy, and J. Zahorjan, "PCP: Efficient endpoint congestion control," in Proceedings of NSDI '06, San Jose, CA, May 2006. 


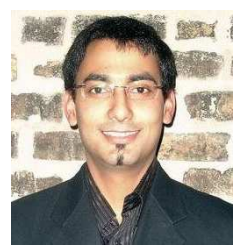

Amit Mondal is a Ph.D. student in the Department of Electrical Engineering and Computer Science at Northwestern University. He received his B.S. degree in computer science and engineering from Indian Institute of Technology, Kanpur, India in 2004. His research interests are in the area of computer networking with emphasis on understanding the behavior of Internet protocols in diverse and extreme scenarios and exploring vulnerabilities in commonly used Internet protocols.

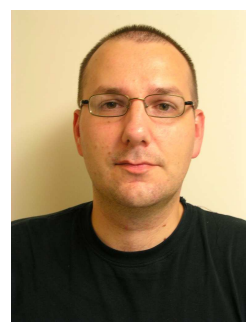

Aleksandar Kuzmanovic is an assistant professor in the Department of Electrical Engineering and Computer Science at Northwestern University. $\mathrm{He}$ received his B.S. and M.S. degrees from the University of Belgrade, Serbia, in 1996 and 1999 respectively. He received the Ph.D. degree from the Rice University in 2004. His research interests are in the area of computer networking with emphasis on design, measurements, analysis, denial-of-service resiliency, and prototype implementation of protocols and algorithms for the Internet. 